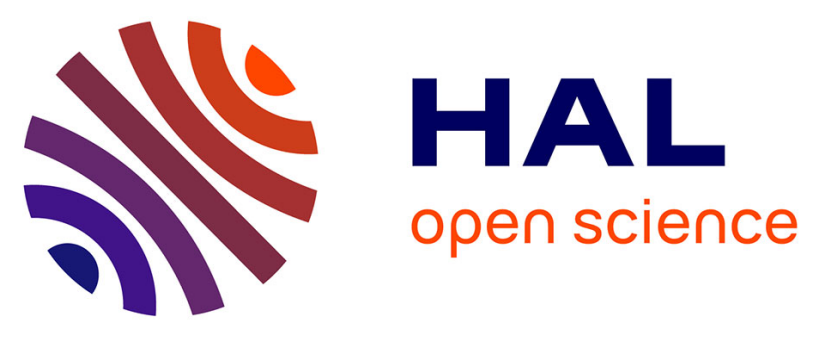

\title{
Synthese von Zinn(I)bromid. Ein neues binäres Halogenid für die Synthesechemie
}

Andreas Schnepf, Claudio Schrenk, Ralf Köppe, Inga Schellenberg, Rainer Pöttgen

\section{- To cite this version:}

Andreas Schnepf, Claudio Schrenk, Ralf Köppe, Inga Schellenberg, Rainer Pöttgen. Synthese von Zinn(I)bromid. Ein neues binäres Halogenid für die Synthesechemie. Journal of Inorganic and General Chemistry / Zeitschrift für anorganische und allgemeine Chemie, 2009, 635 (11), pp.1541. 10.1002/zaac.200900228. hal-00507131

\section{HAL Id: hal-00507131 \\ https://hal.science/hal-00507131}

Submitted on $30 \mathrm{Jul} 2010$

HAL is a multi-disciplinary open access archive for the deposit and dissemination of scientific research documents, whether they are published or not. The documents may come from teaching and research institutions in France or abroad, or from public or private research centers.
L'archive ouverte pluridisciplinaire HAL, est destinée au dépôt et à la diffusion de documents scientifiques de niveau recherche, publiés ou non, émanant des établissements d'enseignement et de recherche français ou étrangers, des laboratoires publics ou privés. 


\section{Synthese von Zinn(I)bromid. Ein neues binäres Halogenid für die Synthesechemie}

\begin{tabular}{|r|l|}
\hline Journal: & Zeitschrift für Anorganische und Allgemeine Chemie \\
\hline Manuscript ID: & zaac.200900228.R1 \\
\hline Wiley - Manuscript type: & Article \\
\hline Complete List of Authors: & $\begin{array}{l}\text { Schnepf, Andreas; University, Inorganic Chemistry } \\
\text { Schrenk, Claudio } \\
\text { Köppe, Ralf } \\
\text { Schellenberg, Inga } \\
\text { Pöttgen, Rainer }\end{array}$ \\
\hline Keywords: & subhalides, tin, thermodynamics, MP2-calculations \\
\hline
\end{tabular}

\section{S ScholaroNE \\ Manuscript Central}


Synthese von Zinn(I)-bromid.

\title{
Ein neues binäres Halogenid für die Synthesechemie
}

\author{
Claudio Schrenk ${ }^{\mathrm{a}}$, Ralf Köppe ${ }^{\mathrm{a}}$, Inga Schellenberg ${ }^{\mathrm{b}}$, Rainer Pöttgen ${ }^{\mathrm{b}}$ und Andreas Schnepf ${ }^{\mathrm{a} *}$ \\ ${ }^{a}$ Karlsruhe, Institut für Anorganische Chemie der Universität \\ ${ }^{\mathrm{b}}$ Münster, Institut für Anorganische und Analytische Chemie der Universität
}

Bei der Redaktion eingegangen am:

\section{Synthesis of tin(I)-bromide.}

\section{A novel binary halide for synthetic chemistry}

Keywords: MP2-Calculations, Tin, Subhalides, Thermodynamics

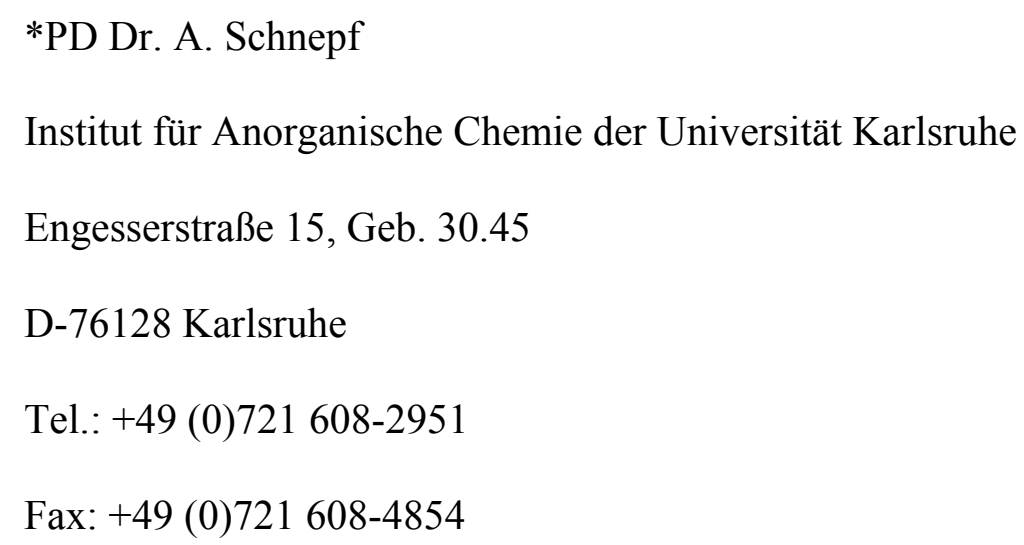

Email: schnepf@chemie.uni-karlsruhe.de 


\begin{abstract}
Thermodynamic data for gaseous $\mathrm{SnBr}$, derived from quantum chemical (MP2)-calculations show that this monohalide should be formed at $1370^{\circ} \mathrm{C}$ as a product of the reaction of elemental tin with $\mathrm{HBr}$ at $1 \cdot 10^{-2}$ mbar. Applying the co-condensation technique $\mathrm{SnBr}$ can be trapped at $-196^{\circ} \mathrm{C}$ and prepared in synthetic scale. Herein first analysis of $\mathrm{SnBr}$ are presented, showing that $\mathrm{SnBr}$ is more reactive than the corresponding $\mathrm{GeBr}$, already disproportionating quantitatively to elemental tin and $\mathrm{SnBr}_{2}$ on heating to room temperature. ${ }^{119} \mathrm{Sn}$ Mössbauer spectroscopic measurements confirm the disproportionation by showing three single signals in the spectra which can be attributed to elemental tin, $\mathrm{SnBr}_{2}$ and a trace amount of $\mathrm{SnO}_{2}$.
\end{abstract}

\title{
Kurztext
}

Thermodynamische Daten für gasförmiges $\mathrm{SnBr}$, welche mit Hilfe quantenchemischer MP2Rechnungen erhalten wurden, zeigen, dass dieses Monohalogenid bei $1370^{\circ} \mathrm{C}$ durch Umsetzung von elementarem Zinn mit $\mathrm{HBr}$ bei einem Druck von 0.01 mbar darstellbar sein sollte. Unter Verwendung der Kokondensationstechnik kann $\mathrm{SnBr}$ im präparativen Maßstab dargestellt und bei $-196^{\circ} \mathrm{C}$ abgefangen werden. Wir beschreiben hier erste Untersuchungen an diesem neuen Monohalogenid, die zeigen, dass $\mathrm{SnBr}$ deutlich reaktiver als $\mathrm{GeBr}$ ist. So disproportioniert $\mathrm{SnBr}$ beim Erwärmen auf Raumtemperatur quantitativ in elementares Zinn und $\mathrm{SnBr}_{2}$. Der quantitative Ablauf der Disproportionierungsreaktion wurde auch durch ${ }^{119} \mathrm{Sn}$ Mößbauerspektroskopie nachgewiesen, wobei drei Signale erhalten werden, die elementarem Zinn, $\mathrm{SnBr}_{2}$ und Spuren von $\mathrm{SnO}_{2}$ zugeordnet werden können. 


\section{Einleitung}

Metalloide Clusterverbindungen des Germaniums der allgemeinen Summenformel $\mathrm{Ge}_{n} \mathrm{R}_{\mathrm{m}}$ $(\mathrm{n}>\mathrm{m})$ konnten innerhalb der letzten Jahre als neue Substanzklasse des Germaniums etabliert werden. ${ }^{1}$ Innerhalb dieser Clusterverbindungen findet man neben ligandgebundenen Germaniumatomen auch solche, die nur an weitere Germaniumatome gebunden sind und denen somit die Oxidationsstufe null zugeordnet werden kann. Da den ligandgebundenen Germaniumatomen die Oxidationsstufe eins zugeordnet werden kann, liegt die mittlere Oxidationsstufe aller Germaniumatome innerhalb einer metalloiden Clusterverbindung zwischen null und eins, d.h. die metalloiden Clusterverbindungen können als molekulare Modellverbindungen für den Grenzbereich zwischen Molekül und Festkörper verstanden werden. $^{2}$

Zur Darstellung metalloider Clusterverbindungen des Germaniums konnten wir innerhalb der letzten Jahre einen erfolgreichen Zugang, ausgehend von Ge(I)-halogeniden wie GeBr oder $\mathrm{GeCl}$ etablieren, wobei metalloide Clusterverbindungen mit bis zu 14 Germaniumatomen im Clusterkern isoliert werden konnten. ${ }^{3}$ Die strukturelle Charakterisierung dieser metalloiden Clusterverbindungen zeigt dabei, dass neuartige Strukturmotive im Bereich zwischen oxidierten molekularen Verbindungen und der Festkörperphase des elementaren Germaniums realisiert werden können. So lässt sich beispielsweise die Anordnung der 10 Germaniumatome in (THF) ${ }_{18} \mathrm{Na}_{6} \mathrm{Ge}_{10}\left[\mathrm{Fe}(\mathrm{CO})_{4}\right]_{8} \mathbf{1}$ als Zentaurpolyeder beschreiben, ${ }^{4}$ ein aus der Festkörperchemie bekanntes Strukturmotiv der Koordinationszahl 10. Im Falle von 1 ist das Polyeder jedoch leer. Außerdem zeigt die Ausbildung eines leeren Polyeders aus 14 Germaniumatomen in $(\mathrm{THF})_{6} \mathrm{Li}_{3} \mathrm{Ge}_{14}\left[\mathrm{Ge}\left(\mathrm{SiMe}_{3}\right)_{3}\right]_{5} \mathbf{2}^{5},{ }^{5}$ dass hier auch größere polyedrische Anordnungen möglich sind, die auf „Germafullerene“ hinweisen. Aufgrund dieser Erfolge im Bereich des Germaniums stellt sich die Frage, ob sich das Synthesekonzept der Darstellung metalloider Clusterverbindungen unter Ausnutzung der Disproportionierungsreaktion von metastabilen Subhalogeniden ${ }^{6}$ auch auf andere Elemente der 14. Gruppe übertragen lässt. 
Dabei ist eine Ausweitung auf das Element Zinn (Schema 1) besonders interessant, da hier aufgrund des deutlich metallischeren Charakters eine größere Strukturvielfalt zu erwarten ist. ${ }^{7}$

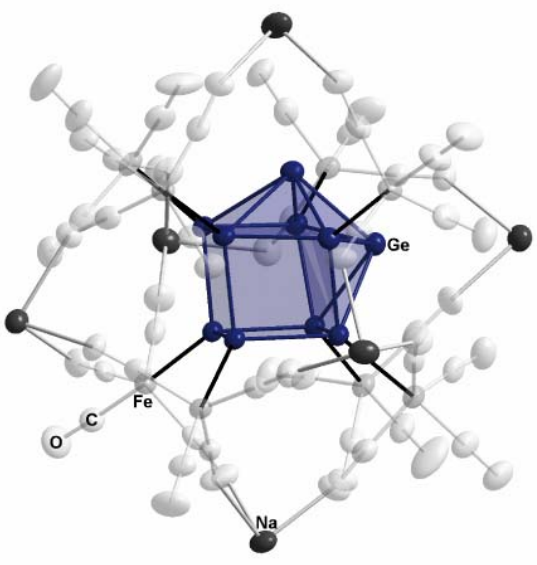

1

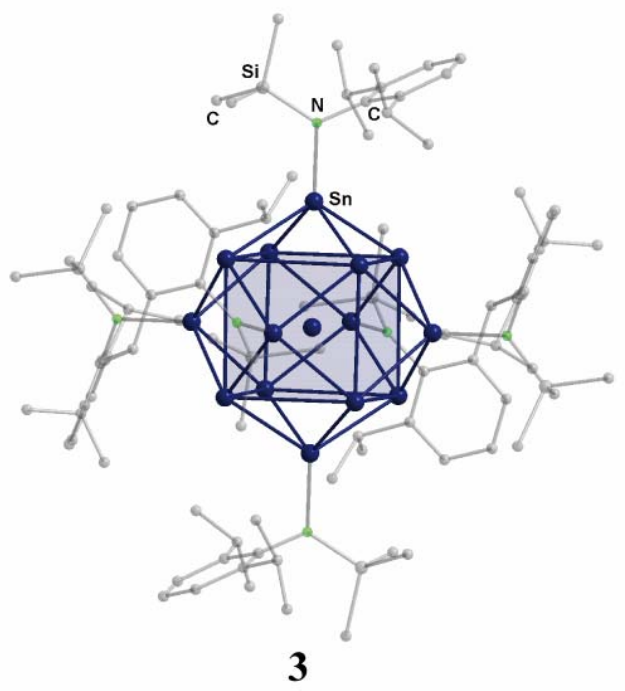

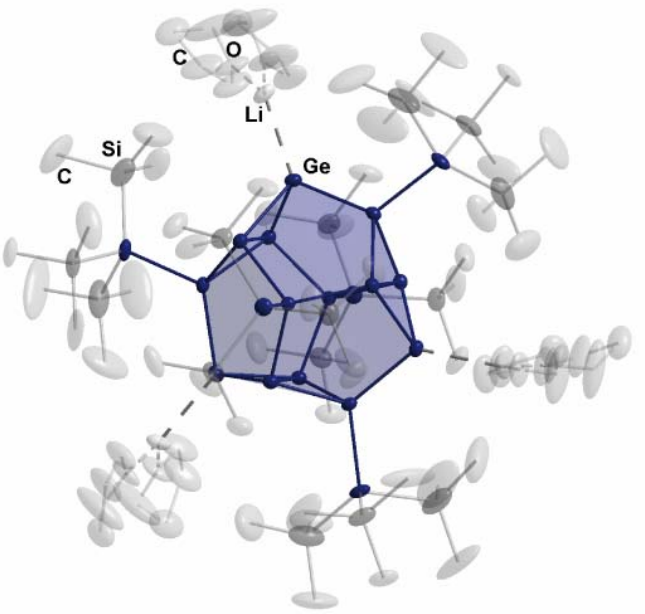

2

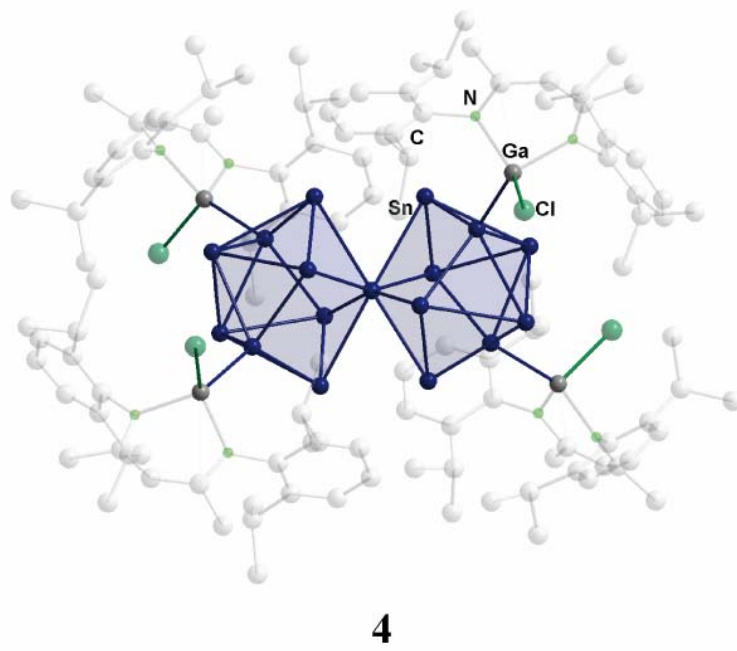

Abbildung1: Molekülstruktur ausgesuchter metalloider Clusterverbindungen des Germaniums (oben) und Zinns (unten) ohne Wasserstoffatome: (THF) ${ }_{18} \mathrm{Na}_{6} \mathrm{Ge}_{10}\left[\mathrm{Fe}(\mathrm{CO})_{4}\right]_{8} \mathbf{1}$ (ohne THF Moleküle), (THF) $)_{6} \mathrm{Li}_{3} \mathrm{Ge}_{14}\left[\mathrm{Ge}\left(\mathrm{SiMe}_{3}\right)_{3}\right]_{5} \mathbf{2}, \mathrm{Sn}_{15} \mathrm{Dipp}_{6} 3\left(\mathrm{Dipp}=\mathrm{NArSiMe} ; \mathrm{Ar}=2,6-i \mathrm{Pr}_{2}-\right.$ $\left.\mathrm{C}_{6} \mathrm{H}_{3}\right)$ und $\mathrm{Sn}_{17}[\mathrm{GaCl}(\mathrm{ddp})]_{4} 4\left(\mathrm{ddp}=\mathrm{HC}(\mathrm{CMeNAr})_{2}\right)$. Die Anordnung der Germanium bzw. Zinnatome im Clusterkern ist durch eine Polyederdarstellung hervorgehoben.

Einige Beispiele metalloider Clusterverbindungen des Zinns konnten innerhalb der letzten Jahre vor allem von den Arbeitsgruppen Power $^{8}$ und Fischer ${ }^{9}$ über die Syntheseroute der 
reduktiven Kupplung erhalten werden, wobei im Falle der metalloiden Clusterverbindung $\operatorname{Sn}_{15}$ Dipp $_{6} 3$ der metallischere Charakter des Zinns zu erkennen ist; ${ }^{8 c}$ so findet man in $\mathbf{3}$ ein zentrales Zinnatom, das nur an acht weitere Zinnatome gebunden ist und das die Koordinationszahl 8+6 der kubisch innenzentrierten Metalle besitzt (Wolfram-Typ).

\section{Ergebnisse und Diskussion}

Zur Entwicklung eines Synthesezugangs $\mathrm{zu}$ metalloiden Zinnclustern über eine Disproportionierungsreaktion (Schema 1) muss als erstes ein geeignetes Ausgangsmaterial (Subhalogenid) zur Verfügung stehen, das schon bei tiefen Temperaturen eine Disproportionierungsreaktion zeigt, damit die intermediär gebildeten Clusterverbindungen $\left(\mathrm{Sn}_{n} \mathrm{Br}_{\mathrm{m}} \mathrm{n}>\mathrm{m}\right)$ kinetisch stabilisiert werden können. Diese kinetische Stabilisierung lässt sich beispielsweise durch Substitution der Halogenatome durch einen sperrigen Liganden realisieren, wodurch der Zinnkern dann durch eine Ligandenhülle nach außen abgeschirmt ist (Schema 1).

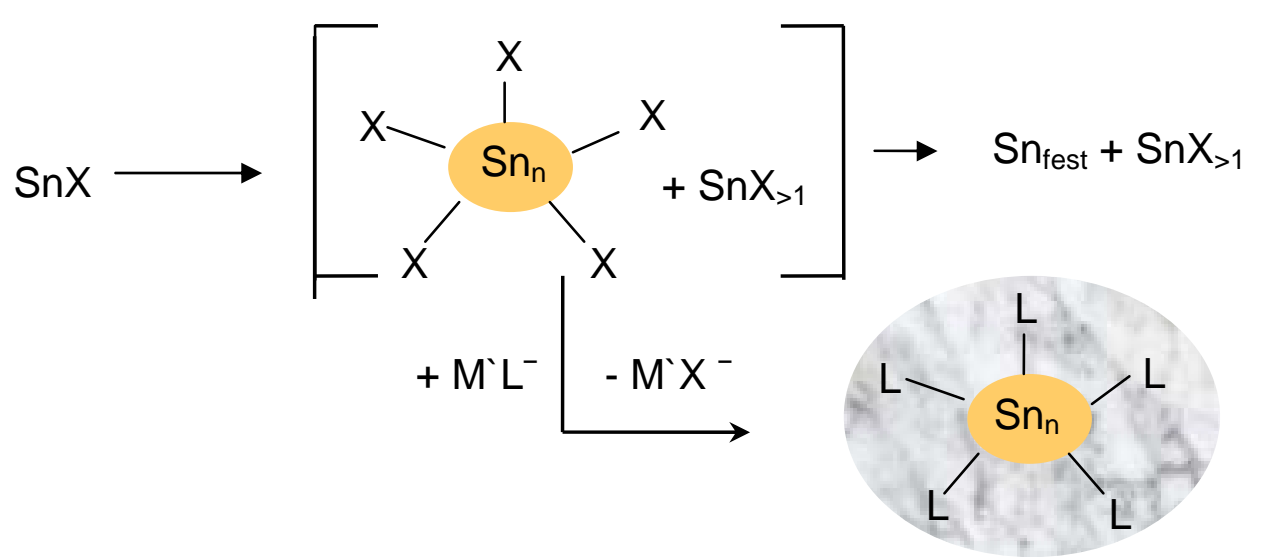

Schema 1: Schematische Darstellung des Synthesekonzeptes zur Darstellung eines metalloiden Zinnclusters über eine Disproportionierungsreaktion eines metastabilen subvalenten Zinnhalogenids. 
Zinn(II)-halogenide scheiden somit als Ausgangsmaterialien für diese Syntheseroute aus, da z.B. $\mathrm{SnBr}_{2}$ bis zum Siedepunkt von $619^{\circ} \mathrm{C}$ gegenüber der Disproportionierung stabil ist. ${ }^{10}$ Zur Entwicklung eines Synthesezugangs zu metalloiden Zinnclustern über die Syntheseroute der Disproportionierungsreaktion, ist es also nötig, einen Schritt weiter auf das Element, d.h. zu den entsprechenden Monohalogeniden zu gehen, welche bisher nur in der Gasphase spektroskopisch untersucht worden sind. ${ }^{11}$

Um zu ermitteln, unter welchen Druck- und Temperaturbedingungen gasförmige Monohalogenide des Zinns in präparativ nutzbaren Ausbeuten $(>50 \%)$ gebildet werden, haben wir eine auf thermodynamischen Daten basierende Abschätzung für die Reaktion $\mathrm{SnBr}_{2(\mathrm{~g})}+\mathrm{Sn}_{(\mathrm{fl} .)} \rightarrow 2 \mathrm{SnBr}_{(\mathrm{g})}$ vorgenommen. Die Partialdrücke einzelner Gaskomponenten bei solchen Hochtemperaturreaktionen können bestimmt werden, wenn die Reaktionstemperaturen ausreichend hoch sind $\left(>700^{\circ} \mathrm{C}\right)$, so dass kinetische Einflüsse unter Knudsen-Bedingungen praktisch bedeutungslos werden. Anhand der Zusammenhänge zwischen den thermodynamischen Werten $\left(\Delta \mathrm{H}, \Delta \mathrm{S}\right.$ und $\left.\mathrm{c}_{\mathrm{p}}\right)$ der an der Reaktion beteiligten Reaktionspartner ist dann die Berechnung der Partialdrücke der einzelnen Gaskomponenten möglich.

Die Geometrien der in Betracht kommenden Gasphasenmoleküle wurden mit quantenchemischen RI-MP2 Rechnungen ${ }^{12}$ unter Verwendung von def2-QZVPP Basissätzen ${ }^{13,14,15,16}$ mit dem Programmpaket TURBOMOLE ${ }^{17}$ berechnet, wodurch sehr gute energetische und spektroskopische Daten erhalten wurden. Die Schwingungsfrequenzen wurden mit dem Modul NUMFORCE durch Diagonalisierung der numerisch berechneten Hesse-Matrix erhalten. Die Entropiewerte und die Wärmekapazitäten wurden über die Zusammenhänge aus der statistischen Thermodynamik ermittelt (Modul FREEH). Die Standardbildungsenthalpie von $\operatorname{SnBr}_{\mathrm{n}}(\mathrm{n}=1,2,4)$ wurde unter Berücksichtigung der theoretisch berechneten Reaktionsenthalpie der Reaktionen (1) bzw. (2): 


$$
\begin{gathered}
\mathrm{Sn}_{\mathrm{g}}+\mathrm{n} \mathrm{HBr} \mathrm{g} \rightarrow \mathrm{SnBr}_{\mathrm{n} g}+\mathrm{n} / 2 \mathrm{H}_{2 \mathrm{~g}} \\
\Delta_{\mathrm{R}} H^{0}{ }_{298}{ }^{\text {calc. }}=\Delta_{\mathrm{f}} H^{0}{ }_{298}{ }^{\text {tot.,calc. }}\left(\mathrm{SnBr}_{\mathrm{n}}\right)+\mathrm{n} / 2 \Delta_{\mathrm{f}} H^{0}{ }_{298}{ }^{\text {tot.,calc. }}\left(\mathrm{H}_{2}\right)-\Delta_{\mathrm{f}} H^{0}{ }_{298}{ }^{\text {tot.,calc. }}(\mathrm{Sn})-\mathrm{n} \Delta_{\mathrm{f}} H^{0}{ }_{298}{ }^{\text {tot.,calc. }}(\mathrm{HBr}) \\
\mathrm{Sn}_{\mathrm{g}}+\mathrm{n} / 2 \mathrm{Br}_{2 \mathrm{~g}} \rightarrow \mathrm{SnBr}_{\mathrm{n} g} \\
\Delta_{\mathrm{R}} H^{0}{ }_{298}{ }^{\text {calc. }}=\Delta_{\mathrm{f}} H^{0}{ }_{298}{ }^{\text {tot.,calc. }}\left(\mathrm{SnBr}_{\mathrm{n}}\right)-\Delta_{\mathrm{f}} H^{0}{ }_{298}{ }^{\text {tot.,calc. }}(\mathrm{Sn})-\mathrm{n} / 2 \Delta_{\mathrm{f}} H^{0}{ }_{298}{ }^{\text {tot.,alc. }}\left(\mathrm{Br}_{2}\right)
\end{gathered}
$$

erhalten. Unter Berücksichtigung der experimentell bestimmten Werte von $\Delta_{\mathrm{f}} H_{298}^{0}$ von $\mathrm{Sn}_{\mathrm{g}}$, $\mathrm{HBr}_{\mathrm{g}}, \mathrm{H}_{2 \mathrm{~g}}$ und $\mathrm{Br}_{2} \mathrm{~g}$ lässt sich die Bildungsenthalpie von $\operatorname{SnBr}_{\mathrm{n}}(\mathrm{n}=1,2,4)$ nach:

$\Delta_{\mathrm{f}} H^{0}{ }_{298}{ }^{\text {calc. }}\left(\mathrm{SnBr}_{\mathrm{n}}\right)=\Delta_{\mathrm{R}} H^{0}{ }_{298}{ }^{\text {calc. }}-\mathrm{n} / 2 \Delta_{\mathrm{f}} H^{0}{ }_{298}{ }^{\text {exp. }}\left(\mathrm{H}_{2}\right)+\Delta_{\mathrm{f}} H^{0}{ }_{298}{ }^{\text {exp. }}(\mathrm{Sn})+\mathrm{n} \Delta_{\mathrm{f}} H^{0}{ }_{298}{ }^{\text {exp. }}(\mathrm{HBr})$

bzW. $\Delta_{\mathrm{f}} H^{0}{ }_{298}{ }^{\text {calc. }}\left(\mathrm{SnBr}_{\mathrm{n}}\right)=\Delta_{\mathrm{R}} H^{0}{ }_{298}{ }^{\text {calc. }}+\Delta_{\mathrm{f}} H^{0}{ }_{298}{ }^{\text {exp. }}(\mathrm{Sn})+\mathrm{n} / 2 \Delta_{\mathrm{f}} H^{0}{ }_{298}{ }^{\text {exp. }}\left(\mathrm{Br}_{2}\right)$

erhalten (Tabelle 1).

Tabelle 1: Thermodynamische Daten der Reaktionen (1) und (2) mit n =1, 2 und 4.

\begin{tabular}{|c|c|c|c|c|c|}
\hline $\mathrm{n}$ & & $\Delta \mathrm{E}_{\mathrm{tot}}{ }^{0 \mathrm{~K}}$ & $\Delta_{\mathrm{r}} H^{0}{ }_{298}$ & $\Delta_{\mathrm{f}} H^{0}{ }_{298}{ }^{\text {theor. }}\left(\mathrm{SnBr}_{\mathrm{n}}\right)$ & $\Delta_{\mathrm{f}} H^{0}{ }_{298}{ }^{\text {exp. }}\left(\mathrm{SnBr}_{\mathrm{n}}\right)^{21}$ \\
\hline 4 & $\mathrm{Sn}+4 \mathrm{HBr}=\mathrm{SnBr}_{4}+2 \mathrm{H}_{2}$ & -531.1 & -532.0 & -376.1 & -347.6 \\
\hline 2 & $\mathrm{Sn}+2 \mathrm{HBr}=\mathrm{SnBr}_{2}+\mathrm{H}_{2}$ & -382.0 & -384.5 & -155.8 & -103.9 \\
\hline 1 & $\mathrm{Sn}+\mathrm{HBr}=\mathrm{SnBr}+1 / 2 \mathrm{H}_{2}$ & -178.8 & -180.7 & 84.4 & 78.5 \\
\hline 4 & $\mathrm{Sn}+2 \mathrm{Br}_{2}=\mathrm{SnBr}_{4}$ & -751.9 & -747.6 & -384.3 & -347.6 \\
\hline 2 & $\mathrm{Sn}+\mathrm{Br}_{2}=\mathrm{SnBr}_{2}$ & -492.4 & -492.3 & -159.9 & -103.9 \\
\hline 1 & $\mathrm{Sn}+1 / 2 \mathrm{Br}_{2}=\mathrm{SnBr}$ & -234.0 & -234.6 & 82.3 & 78.5 \\
\hline
\end{tabular}

Der Vergleich der berechneten Enthalpiewerte von $\mathrm{SnBr}_{2}$ und $\mathrm{SnBr}_{4}$ mit den experimentell ermittelten zeigt eine Unsicherheit etwa $\pm 40 \mathrm{~kJ} / \mathrm{mol}$. Dies ist in zufriedenstellender Übereinstimmung mit anderen Berechnungen kleiner Moleküle. ${ }^{18}$ Die Übereinstimmung der berechneten Standardbildungsenthalpie von $\mathrm{SnBr}$ mit dem von Gingerich erhaltenen experimentellen Wert ist mit $\pm 6 \mathrm{~kJ} / \mathrm{mol}$ noch deutlich besser. ${ }^{19}$ Die berechneten Entropiewerte entsprechen wegen der hohen Güte der theoretischen Schwingungsfrequenzen praktisch exakt den experimentell erhaltenen Werten. Die Temperaturabhängigkeit von $\Delta_{\mathrm{f}} \mathrm{H}^{0}{ }_{\mathrm{T}}$ und $\mathrm{S}_{\mathrm{T}}^{0}$ ist durch das Kirchhoffsche Gesetz gegeben ${ }^{21}$. Die Wärmekapazität $\mathrm{c}_{\mathrm{p}}{ }_{\mathrm{T}}$ wird durch das folgende Polynom ausgedrückt: $\mathrm{c}_{\mathrm{p}}{ }^{0} \mathrm{~T}=\mathrm{a}+\mathrm{b} 10^{-3} \mathrm{~T}+\mathrm{c} 10^{6} \mathrm{~T}^{-2}+\mathrm{d} 10^{-6} \mathrm{~T}^{2}$. Die Koeffizienten für $\mathrm{c}_{\mathrm{p}}{ }^{0} \mathrm{~T}$ 
von $\mathrm{SnBr}$ wurden mit dem Mathematikprogramm MAPLE ${ }^{20}$ erhalten, wobei vier theoretisch berechnete Werte für $\mathrm{c}_{\mathrm{p}}{ }^{0} \mathrm{~T}(\mathrm{~T}=298.15,1032.1,1766.05,2500 \mathrm{~K}$; Modul FREEH), verwendet werden.

Tabelle 2: Verwendete thermochemische Daten zur Berechnung der Partialdrücke der gasförmigen Komponenten im Reaktionssystem flüssiges Zinn / HBr.

\begin{tabular}{|c|c|c|c|c|c|c|c|}
\hline & $\begin{array}{l}\Delta_{\mathrm{f}} \mathrm{H}^{0}{ }_{298} \\
{\left[\mathrm{~kJ} \mathrm{~mol}^{-1}\right]}\end{array}$ & $\begin{array}{l}\mathrm{S}_{298}^{0} \\
{\left[\mathrm{~J} \mathrm{~mol}^{-1} \mathrm{~K}^{-1}\right]}\end{array}$ & $\begin{array}{l}\mathrm{c}_{\mathrm{p} \mathrm{T}}^{0}=\mathrm{a} \\
{\left[\mathrm{J} \mathrm{mol}^{-1}\right]}\end{array}$ & $\begin{array}{l}\text { b } 10^{-3} \\
1]\end{array}$ & c $10^{6}$ & $+\mathrm{d} 10^{-6} \mathrm{~T}^{2}$ & Lit. \\
\hline & & & $a$ & $\mathrm{~b}$ & $\mathrm{c}$ & $\mathrm{d}$ & \\
\hline $\mathrm{H}_{2}$ & 0 & 130.7 & 26.88 & 3.59 & 0.11 & 0 & 21 \\
\hline $\mathrm{HBr}$ & -36.4 & 198.7 & 27.53 & 4.59 & -0.01 & 0 & 21 \\
\hline $\operatorname{Sn}_{(1 .)}$ & 13 & 80.2 & 21.69 & 6.15 & 1.29 & 0 & 21 \\
\hline $\mathrm{SnBr}_{2}$ & -103.9 & 335.8 & 58.14 & 1.34 & -0.3 & 0 & 21 \\
\hline $\mathrm{SnBr}_{4}$ & -347.6 & 412.4 & 107.51 & 0.64 & -0.39 & 0 & 21 \\
\hline $\mathrm{SnBr}$ & 84.4 & 258.8 & 37.37 & 0.05 & -0.08 & -0.01 & diese Arbeit \\
\hline $\mathrm{Br}$ & 111.9 & 175 & 19.87 & 1.49 & 0.04 & 0 & 21 \\
\hline $\mathrm{Br}_{2}$ & 30.9 & 245.4 & 37.36 & 0.46 & -1.3 & 0 & 21 \\
\hline
\end{tabular}

Unter Verwendung der so erhaltenen thermodynamischen Daten von $\mathrm{SnBr}$ und der experimentell bestimmten Werte von $\mathrm{Sn}_{\mathrm{fl}}, \mathrm{SnBr}_{2}, \mathrm{SnBr}_{4}, \mathrm{HBr}, \mathrm{H}_{2}$ und $\mathrm{Br}_{2}$ (Tabelle 2) wurden die Partialdrücke der einzelnen Verbindungen im thermodynamischen Gleichgewicht nach einem von Binnewies beschriebenen Verfahren iterativ bestimmt, ${ }^{22}$ wobei die Reaktionen von Zinn mit $\mathrm{HBr}$ unter Bildung von $\mathrm{SnBr}_{4}, \mathrm{SnBr}_{2}$ bzw. $\mathrm{SnBr}$, der Zerfall von $\mathrm{HBr} \mathrm{zu} \mathrm{H}_{2}$ und $\mathrm{Br}_{2}$, sowie die thermische Dissoziation von $\mathrm{Br}_{2}$ in Bromatome zugrunde gelegt wurden:

$$
\begin{array}{clll}
4 \mathrm{HBr}_{\mathrm{g}}+\mathrm{Sn} . & \rightarrow \mathrm{SnBr}_{4(\mathrm{~g})}+2 \mathrm{H}_{2} & \mathrm{a} \\
2 \mathrm{HBr}_{\mathrm{g}}+\mathrm{Sn} . & \rightarrow & \mathrm{SnBr}_{2(\mathrm{~g})}+\mathrm{H}_{2} & \mathrm{~b} \\
\mathrm{HBr}_{\mathrm{g}}+\mathrm{Sn} & \rightarrow & \mathrm{SnBr}_{(\mathrm{g})}+1 / 2 \mathrm{H}_{2} & \mathrm{c} \\
2 \mathrm{HBr}_{\mathrm{g}} & \rightarrow & \mathrm{Br}_{2}+\mathrm{H}_{2} & \mathrm{~d} \\
\mathrm{Br}_{2} & \rightarrow & & \mathrm{e}
\end{array}
$$


Außerdem wurden die Bestimmungsgleichungen $\mathrm{p}_{0}(\mathrm{HBr})=\mathrm{p}(\mathrm{HBr})+2 \mathrm{p}\left(\mathrm{H}_{2}\right)$ und $\mathrm{p}_{0}(\mathrm{HBr})=$ $\mathrm{p}(\mathrm{HBr})+2 \mathrm{p}\left(\mathrm{Br}_{2}\right)+\mathrm{p}(\mathrm{SnBr})+2 \mathrm{p}\left(\mathrm{SnBr}_{2}\right)+4 \mathrm{p}\left(\mathrm{SnBr}_{4}\right)+\mathrm{p}(\mathrm{Br})$ berücksichtigt, bei denen $\mathrm{p}_{0}(\mathrm{HBr})$ dem HBr Partialdruck vor Einstellung des Gleichgewichtes entspricht. Das Ergebnis dieser Rechnung ist in Abbildung 2 für einen $\mathrm{HBr}$ Anfangsdruck von $10^{-2}$ mbar angegeben. $^{23}$

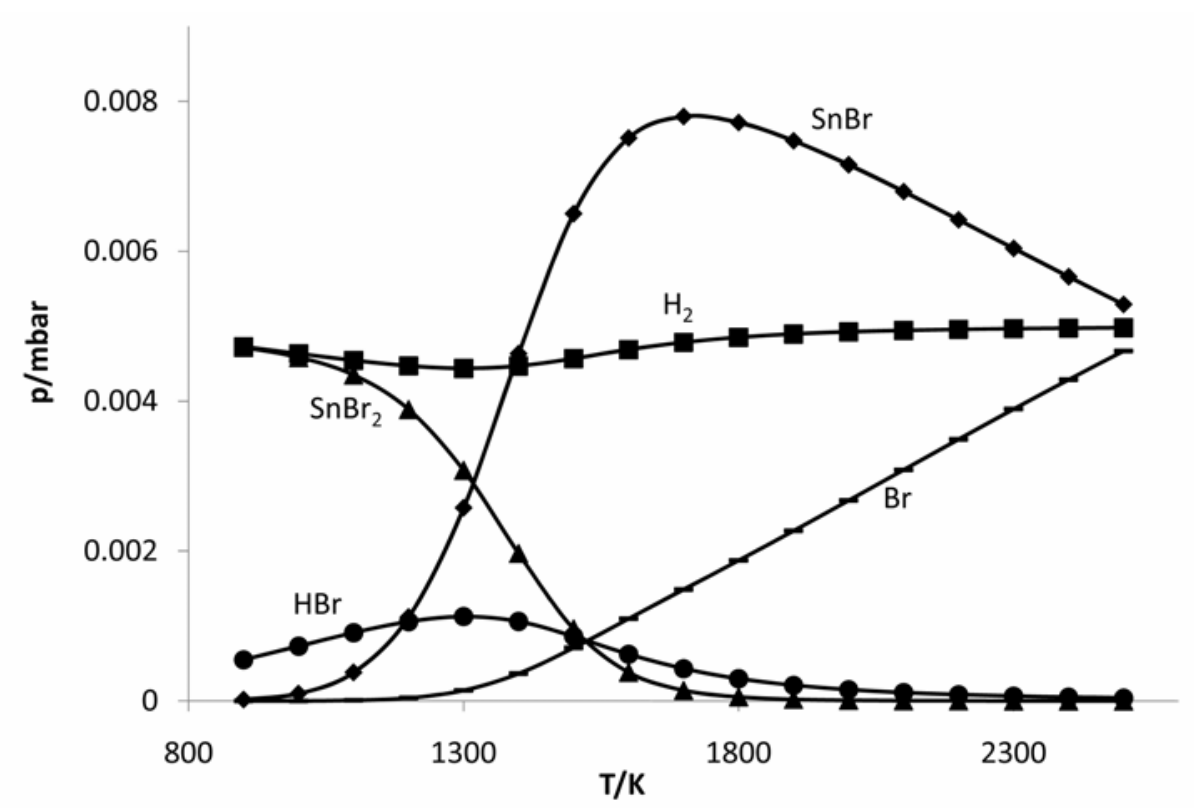

Abbildung 2: Temperaturverlauf der Partialdrucke gasförmiger Verbindungen im

Reaktionssystem flüssiges Zinn / $\mathrm{HBr}\left(\mathrm{p}_{0}(\mathrm{HBr})=0.01\right.$ mbar). (Die Partialdrücke von $\mathrm{SnBr}_{4}$ und $\mathrm{Br}_{2}$ sind nicht aufgeführt, da ihre Werte für jede Temperatur kleiner als $10^{-6}$ mbar sind.)

Wie in Abbildung $2 \mathrm{zu}$ erkennen ist, sollte der maximale Anteil an $\mathrm{SnBr}$ bei einer Reaktionstemperatur im Bereich von $1650 \mathrm{~K}$ erreicht werden. Bei dieser Temperatur erhält man einen 33fachen Überschuss an $\mathrm{SnBr}$ gegenüber $\mathrm{SnBr}_{2}$. Zur Darstellung der Monohalogenide des Zinns im präparativen Maßstab bietet sich die präparative Kokondensationstechnik an, welche wir in einer selbst gebauten Anlage durchführen. ${ }^{24}$

Dabei wird elementares Zinn in einem Graphitreaktor vorgelegt, der induktiv bis $2000^{\circ} \mathrm{C}$ geheizt werden kann. Bei der Versuchsdurchführung wird über das heiße flüssige Zinn (ca. $1240^{\circ} \mathrm{C}$ ), bei reduziertem Druck (ca. 0.01 mbar) $\mathrm{HBr}$ geleitet und die bei der Reaktion 
entstehenden gasförmigen Teilchen werden zusammen mit einem zudosierten Lösungsmittel bei $-196^{\circ} \mathrm{C}$ ausgefroren. Gleichzeitig wird der entstehende Wasserstoff kontinuierlich über ein Hochvakuumpumpensystem abgepumpt, so dass der Druck in der Anlage während des Versuchs im Bereich von $5 \cdot 10^{-5}$ mbar gehalten wird. Nach beendeter Reaktion wird die Stickstoffkühlung entfernt, und die Anlage wird mit gasförmigem Stickstoff geflutet. Beim Erwärmen schmilzt die gebildete Matrix, und die entstehende Lösung kann über eine Stahlkanüle mit Stickstoffüberdruck in eine gekühlte Vorlage überführt werden.

Setzt man so Zinn mit $\mathrm{HBr}$ bei $1240^{\circ} \mathrm{C}$ um, und kondensiert die bei der Reaktion entstehenden gasförmigen Teilchen mit Toluol, so erhält man als Produkt eine farblose Lösung, in der ein schwarzer Feststoff suspendiert ist, der in dünnen Schichten violett erscheint. Bei der Reaktion wurden bei 20 mmol eingesetztem $\mathrm{HBr} 17$ mmol Zinn verbraucht, was einem Verbrauchsverhältnis (Zinn zu Halogen) von 1:1.176 entspricht. Dies spricht für eine annähernd quantitative Bildung von $\mathrm{SnBr}$ und steht im Rahmen der Fehlergrenzen im Einklang mit den Ergebnissen der o.a. Abschätzung. ${ }^{25}$ Dieses Ergebnis zeigt, dass SnBr durch eine Kokondensationstechnik in präparativen Mengen zugänglich ist.

Zur weiteren Analyse wird die Lösung abfiltriert und der Rückstand am Ölpumpenvakuum getrocknet, wobei er langsam auf Raumtemperatur erwärmt wird. Man erhält dabei einen dunkelgrauen Feststoff 5, dessen Halogengehalt laut Titration eine formale Zusammensetzung SnBr ergibt. Das Pulverdiffraktogramm des Rückstandes zeigte jedoch, dass im Feststoff 5 sowohl elementares Zinn als auch $\mathrm{SnBr}_{2}$ als kristalline Spezies enthalten sind. Weiterhin zeigt auch das Mößbauerspektrum des Rückstandes (Abbildung 3) ein deutliches Signal für elementares Zinn, wie am Vergleichsspektrum zu erkennen ist.

Das Signal mit einer Isomerieverschiebung von $4.07 \mathrm{~mm} / \mathrm{s}$, lässt sich anhand von Literaturdaten $\left(\delta 3.92, \Gamma 1.40, \Delta E_{\mathrm{Q}} 0.0\right)$ eindeutig als $\mathrm{SnBr}_{2}$ identifizieren. ${ }^{26,27}$ Außerdem ist noch ein kleines Signal bei einer Isomerieverschiebung von $0.08 \mathrm{~mm} / \mathrm{s} \mathrm{zu}$ erkennen, was 
eindeutig $\mathrm{SnO}_{2}$ zugeordnet werden kann, ${ }^{28}$ welches sehr wahrscheinlich beim Abschmelzen aus $\mathrm{Sn}$ und $\mathrm{SiO}_{2}$ entstanden ist.

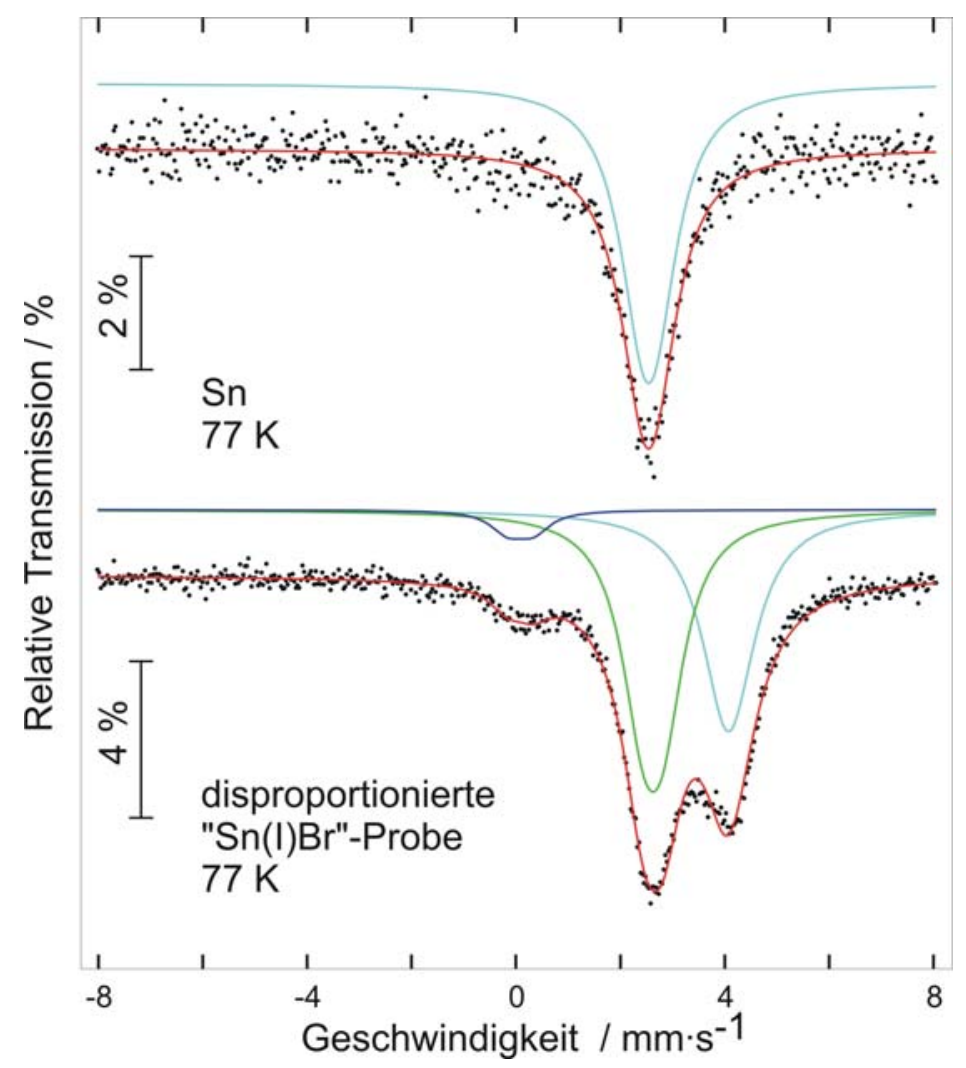

\footnotetext{
Abbildung 3: Experimentelle und simulierte ${ }^{119}$ Sn-Mößbauer-Spektren von $\mathbf{5}$ und elementarem Zinn bei $77 \mathrm{~K}$.
}

Die Intensität der Signale von $\mathrm{SnBr}_{2}$ und $\mathrm{Sn}$ liefert ein Verhältnis von 1:1.05, d.h. beide Spezies liegen im gleichen Mengenverhältnis vor, was eindeutig auf den quantitativen Ablauf einer Disproportionierungsreaktion $\left(2 \mathrm{SnBr} \rightarrow \mathrm{Sn}+\mathrm{SnBr}_{2}\right)$ hindeutet.

Da der Rückstand bei $-78{ }^{\circ} \mathrm{C}$ einen violetten Farbton besitzt, könnte bei dieser Temperatur noch ein Anteil an $\mathrm{SnBr}$ vorliegen, welches dann bei Erwärmen auf Raumtemperatur quantitativ in elementares $\mathrm{Zinn}$ und $\mathrm{SnBr}_{2}$ disproportioniert. Somit ist $\mathrm{SnBr}$ deutlich reaktiver als $\mathrm{GeBr}$, welches in fester Form erst $\mathrm{ab} 90^{\circ} \mathrm{C}$ in $\mathrm{Ge}$ und $\mathrm{GeBr}_{4}$ disproportioniert.

Um zu klären, ob sich die höhere Reaktivität für Folgereaktionen nutzen lässt bzw. ob sie sich reduzieren lässt, um $\mathrm{SnBr}$ als Reagenz zugänglich $\mathrm{zu}$ machen, haben wir im Folgenden 
weitere Kokondensationsreaktionen mit verschiedenen Lösungsmitteln bzw. Donormolekülen durchgeführt: Bei einer Kokondensation von $\mathrm{SnBr}$ mit Aceton erhält man als Produkt eine schwarze Suspension aus einer klaren Lösung und einem schwarzen Feststoff. Nach Aufarbeitung der klaren Lösung erhält man bei $-28^{\circ} \mathrm{C}$ farblose nadelförmige Kristalle von $\mathrm{SnBr}_{2} \cdot 2 \mathrm{OC}_{3} \mathrm{H}_{6}$ 6, die beim Erwärmen auf Raumtemperatur schnell eintrüben. Die Molekülstruktur von 6 ist in Abbildung 4 angegeben und man erkennt ein zentrales Zinnatom, das an zwei Bromatome mit einem mittleren Sn-Br Abstand von 264.3 pm gebunden ist und das weiterhin an zwei Acetonmoleküle mit einem Sn-O Abstand von 253.8 pm gebunden ist. Im Festkörper bilden sich weiterhin $\mathrm{Sn}-\mathrm{Br}$ Kontakte zu benachbarten $\mathrm{SnBr}_{2}$ Molekülen (SnBr: $363.4 \mathrm{pm}$ ) aus, so dass eine verzerrt oktaedrische Koordination der Zinnatome erhalten wird. Das ${ }^{119}$ Sn-NMR Spektrum von aufgelösten Kristallen von 6 zeigt ein scharfes Signal bei $\delta=-68 \mathrm{ppm}$, was dem normalen Bereich zweiwertiger Zinnverbindungen entspricht. ${ }^{29} \mathrm{Im}$ schwarzen Reaktionsrückstand lassen sich über eine Halogenidtitration nur geringe Mengen Bromid bestimmen, was darauf hindeutet, dass es sich dabei um elementares Zinn handelt. Dieser Befund zeigt, dass $\mathrm{SnBr}$ nicht mit Aceton reagiert, sondern dass die Disproportionierungsreaktion bevorzugt abläuft. Dieser Befund steht im Gegensatz zur Situation bei Monohalogeniden des Germaniums, die mit Aceton in einer Redoxreaktion reagieren, ${ }^{30}$ d.h. die Disproportionierungsreaktion ist im Falle der $\mathrm{Sn}(\mathrm{I})$-halogenide deutlich bevorzugt. Um die hohe Reaktivität der Sn(I)-halogenide zu senken, haben wir im Folgenden Kokondensationsreaktionen mit Donormolekülen durchgeführt. Bei einer Kokondensationsreaktion mit einer Mischung aus THF und Toluol im Verhältnis 1:4 erhält man wiederum eine schwarze Suspension aus einer farblosen Lösung und einem schwarzen Rückstand, in dem wiederum kein Halogenid nachweisbar ist. Aus der Lösung lässt sich nach Aufarbeiten die Verbindung $\mathrm{SnBr}_{2} \cdot 2 \mathrm{OC}_{4} \mathrm{H}_{8} 7$ in Form farbloser Nadeln erhalten. Im Festkörper von 7 liegen wie im Falle von $6 \mathrm{SnBr}_{2}$ Moleküle vor ( $\mathrm{Sn}-\mathrm{Br}$ Abstand: 262.2 pm), 
die an zwei THF Moleküle mit einem mittleren Sn-O Abstand von 250.0 pm gebunden sind (Abbildung 5).
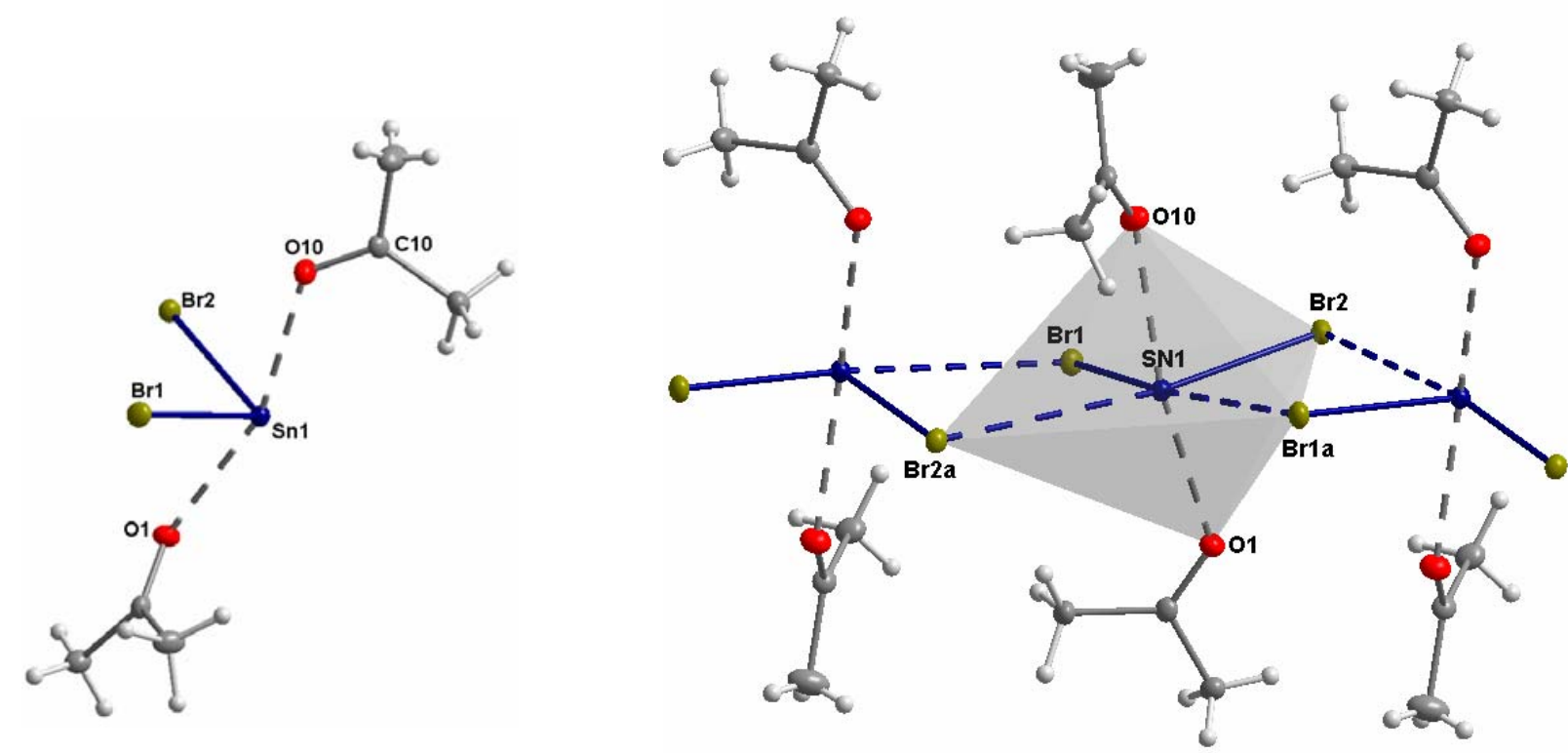

Abbildung 4: Links: Molekülstruktur von $\mathrm{SnBr}_{2} \cdot 2 \mathrm{OC}_{3} \mathrm{H}_{6} 6$ (Schwingungsellipsoide 25\% Wahrscheinlichkeit). Rechts: Erste Koordinationssphäre des Zinnatoms im Kristall; die verzerrt oktaedrische Koordination ist durch eine Polyederdarstellung hervorgehoben. Ausgesuchte Bindungslängen [pm] und Winkel [ $\left.{ }^{\circ}\right]$ : Sn1-Br1: 264.78(7), Sn1-Br2: 263.86(7), Sn1-O1: 255.4(4); Sn1-O10: 252.2(4), Sn1-Br1a: 369.3(1), Sn1-Br2a: 357.5(1), O10-C10: 121.5(7), Br1-Sn1-Br2: 94.08(2), O1-Sn1-O10: 161.35(13), Br1a-Sn1-Br2a: 99.49(2).

Außerdem werden zusätzlich noch zwei lange Sn-Br Kontakte mit einem mittleren Abstand von $393 \mathrm{pm}$ zu benachbarten $\mathrm{SnBr}_{2}$ Molekülen ausgebildet, so dass sich für das Zinnatom wiederum eine verzerrt oktaedrische Koordinationsumgebung ergibt. Sauerstoffhaltige Donormoleküle wie Aceton oder THF scheinen somit nicht geeignet zu sein, um $\operatorname{Sn}(\mathrm{I})-$ halogenide zu stabilisieren, so dass diese für weitere Untersuchungen verwendet werden können. 

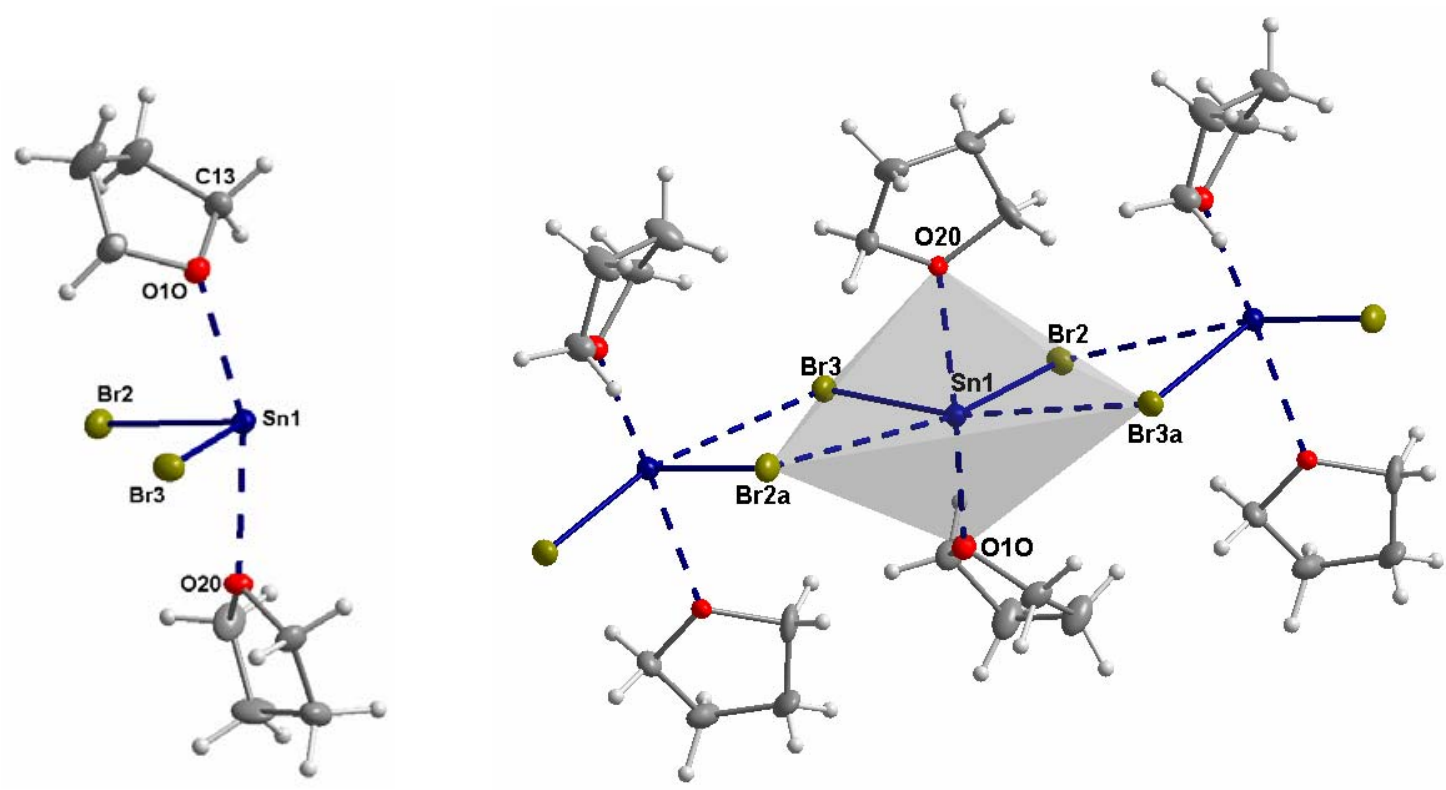

Abbildung 5: Links: Molekülstruktur von $\mathrm{SnBr}_{2} \cdot 2 \mathrm{OC}_{4} \mathrm{H}_{8} 7$ (Schwingungsellipsoide 25\% Wahrscheinlichkeit). Rechts: Erste Koordinationssphäre des Zinnatoms im Kristall; die verzerrt oktaedrische Koordination ist durch eine Polyederdarstellung hervorgehoben. Ausgesuchte Bindungslängen [pm] und Winkel [ $\left.{ }^{\circ}\right]$ : Sn1-Br2: 262.47(5), Sn1-Br3: 261.89(5), Sn1-O10: 249.6(3); Sn1-O20: 250(2), Sn1-Br2a: 403.6(1), Sn1-Br3a: 382.0(1), O10-C13: 146.0(5), Br2-Sn1-Br3: 96.097(18), O10-Sn1-O20: 161.0(4), Br2a-Sn1-Br3a: 107.87(2).

Aus diesem Grund haben wir im Folgenden stickstoffhaltige Donoren wie $\mathrm{N}^{n} \mathrm{Bu}_{3}$ verwendet, die schon im Falle der Ge(I)-halogenide zu metastabilen Emulsionen geführt haben. Auch im Falle der $\mathrm{Sn}(\mathrm{I})$-halogenide führt die Kokondensationsreaktion von $\mathrm{Sn}(\mathrm{I}) \mathrm{Br}$ mit einer Mischung aus Toluol und $\mathrm{N}^{n} \mathrm{Bu}_{3}$ im Verhältnis 4:1 zur Bildung einer öligen Phase. Da die Erhöhung der Dielektrizitätskonstanten der Lösung im Falle des Germaniums zu isolierbaren Lösungen geführt hat, ${ }^{4}$ haben wir im Folgenden eine Kokondensationsreaktion von Sn mit einer Mischung aus $\mathrm{CH}_{2} \mathrm{Cl}_{2}$ und $\mathrm{N}^{n} \mathrm{Bu}_{3}$ im Verhältnis 4:1 durchgeführt, wobei nach beendeter Kokondensation und Erwärmen des Kokondensates auf $-78^{\circ} \mathrm{C}$ eine dunkelbraune Lösung erhalten wurde.

Erwärmt man diese dunkelbraune Lösung auf Raumtemperatur, fällt ein schwarzer Feststoff aus (Abbildung 6) und es bleibt eine blassgelbe Lösung zurück, was auf das Ablaufen einer 
Disproportionierungsreaktion hindeutet. Diese Annahme wird durch das Ergebnis einer Röntgenfluoreszenzanalyse des Rückstandes gestützt, in dem ein Zinn/Halogen Verhältnis $($ Halogen $=\mathrm{Br}$ und $\mathrm{Cl})$ von 6,04:1 gefunden wird. Die im Rückstand gefundenen Mengen an Chlorid deuten schon auf eine Folgereaktion mit dem Lösungsmittel hin. Unglücklicherweise ist $\mathrm{Sn}(\mathrm{I}) \mathrm{Br}$ selbst bei $-78^{\circ} \mathrm{C}$ in der Mischung aus $\mathrm{CH}_{2} \mathrm{Cl}_{2}$ und $\mathrm{N}^{n} \mathrm{Bu}_{3}$ nicht stabil: So entfärbt sich die Lösung innerhalb von zwei Wochen, und man erhält eine blassgelbe Lösung, aus der beim Erwärmen auf Raumtemperatur auch kein schwarzer Feststoff mehr ausfällt. Nach Entfernen des Lösungsmittels erhält man nun einen blassgelben Feststoff (Abbildung 6).

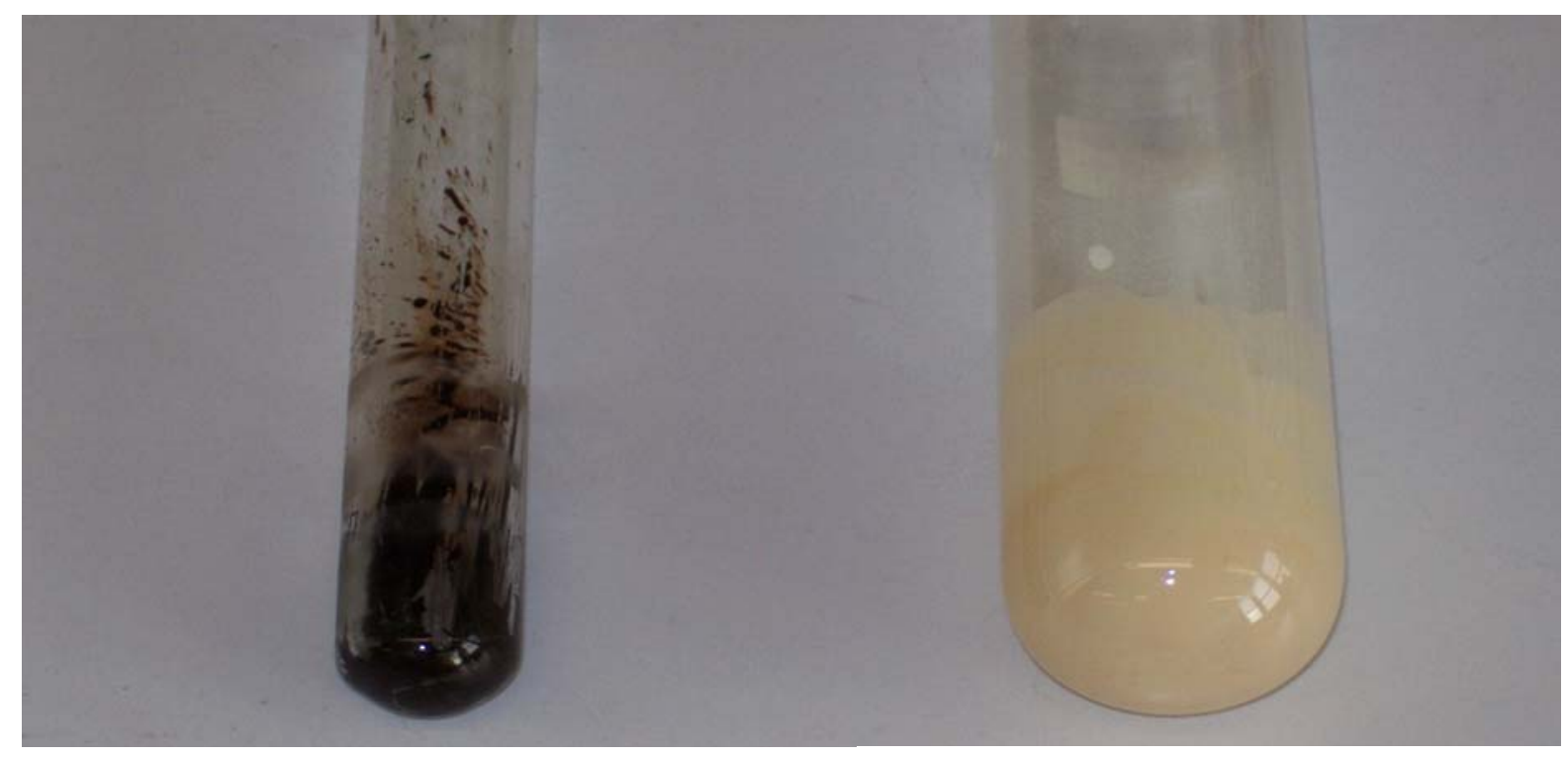

Abbildung 6: Links: Rückstand nach Erwärmen der $\mathrm{SnBr}$ Lösung in $\mathrm{CH}_{2} \mathrm{Cl}_{2} / \mathrm{N}^{n} \mathrm{Bu}_{3}$ direkt nach der Kokondensation. Rechts: Rückstand nach zwei Wochen Lagerung bei $-78^{\circ} \mathrm{C}$ und Entfernen des Lösungsmittels.

\section{Zusammenfassung und Ausblick}

Nach einer thermodynamischen Abschätzung sollte gasförmiges $\mathrm{Sn}(\mathrm{I}) \mathrm{Br}$ in maximalen Ausbeuten bei einer Reaktionstemperatur von $1377^{\circ} \mathrm{C}$ und einem Reaktionsdruck von 0.01 mbar im Reaktionssystem flüssiges Zinn / $\mathrm{HBr}$ darstellbar sein. Die Synthese von $\mathrm{SnBr}$ im präparativen Maßstab erfolgt über die präparative Kokondensation, wobei der Verbrauch an Zinn und $\mathrm{HBr}$ während der Hochtemperaturreaktion auf die Bildung von reinem $\mathrm{SnBr}$ 
hindeutet. Abgeschrecktes $\mathrm{SnBr}$ ist jedoch gegenüber $\mathrm{GeBr}$ deutlich reaktiver und disproportioniert schon bis Raumtemperatur quantitativ in elementares Zinn und $\mathrm{SnBr}_{2}$. Die höhere Reaktivität zeigt sich auch gegenüber einer Mischung aus $\mathrm{CH}_{2} \mathrm{Cl}_{2}$ und $\mathrm{N}^{n} \mathrm{Bu}_{3}$, mit der $\mathrm{SnBr}$ schon bei $-78^{\circ} \mathrm{C}$ abreagiert, wohingegen eine $\mathrm{GeBr}$ Lösung im selben Lösungsmittelgemisch über Monate bei $-78^{\circ} \mathrm{C}$ lagerbar ist. ${ }^{31}$ Zukünftige Untersuchungen konzentrieren sich vor allem auf eine bessere Stabilisierung von SnBr, d.h. auf die Darstellung handhabbarer $\mathrm{SnBr}$ Lösungen. Außerdem sind wir momentan dabei zu untersuchen, ob sich die bei der Kokondensation von $\mathrm{SnBr}$ mit einer Mischung aus Toluol und $\mathrm{N}^{n} \mathrm{Bu}_{3}$ erhaltene Emulsion für Folgereaktionen zur Darstellung metalloider Zinncluster eignet, da dieser Weg im Falle der Monohalogenide des Germaniums erfolgreich etabliert wurde.

\section{Experimentelles}

Die Versuchsdurchführung erfolgt in der in Literatur 24 beschriebenen Anlage, welche über eine Öldiffusionspumpe (Balzers Diff 900) auf einen Druck von $5 \cdot 10^{-6}$ mbar evakuiert wird. In dem im Zentrum der Anlage befindlichen Graphitreaktor wird elementares Zinn vorgelegt. Der Graphitreaktor wird anschließend induktiv über ein hochfrequentes Wechselfeld von $1000 \mathrm{kHz}$ auf $1240^{\circ} \mathrm{C}$ erhitzt. Nachdem der Graphitreaktor eine halbe Stunde im Vakuum ausgeheizt wurde, wird die Edelstahlglocke mit flüssigem Stickstoff auf $-196^{\circ} \mathrm{C}$ gekühlt. Anschließend wird über das $1240^{\circ} \mathrm{C}$ heiße Zinn ein Volumenstrom an $\mathrm{HBr}$ geleitet, wobei die Menge an $\mathrm{HBr}$ durch den Druckabfall in einem Vorratskolben über ein Differenzdruckmanometer (MKS Instruments Inc.; Bereich 100 mbar) bestimmt wird. Der Volumenstrom an $\mathrm{HBr}$ wird hierbei so gewählt $(0.2 \mathrm{mmol} / \mathrm{min})$, dass der Druck in der Anlage während des Versuches ca. $5 \cdot 10^{-5}$ mbar beträgt. Während des Einleitens des $\mathrm{HBr}$ Volumenstroms wird gleichzeitig ein Lösungsmittel über den Lösungsmitteleinlass zudosiert. 
Nachdem im Laufe von 2 Stunden $20 \mathrm{mmol} \mathrm{HBr}$ verbraucht wurden, wobei gleichzeitig ca. $150 \mathrm{ml}$ Lösungsmittel einkondensiert wurden, wird die Ofenheizung abgeschaltet, und die Anlage wird nach dem Entfernen der Flüssigstickstoffkühlung mit Stickstoff 4.8 geflutet. Die Edelstahlglocke wird anschließend mit Trockeneis auf $-78^{\circ} \mathrm{C}$ erwärmt. Nach dem Schmelzen der Matrix wird das Lösungsmittel mit Stickstoffüberdruck in ein mit Trockeneis gekühltes Schlenkgefäß überführt.

\section{Synthese von $\mathrm{SnBr}_{2} \cdot 2 \mathrm{OC}_{3} \mathrm{H}_{6} 6$}

Es werden innerhalb von 2 Stunden $20 \mathrm{mmol}$ Bromwasserstoff bei $1240^{\circ} \mathrm{C}$ über metallisches Zinn geleitet, wobei $2.29 \mathrm{~g}(19 \mathrm{mmol})$ Zinn verbraucht werden. Die bei der Reaktion entstandenen gasförmigen Teilchen werden gemeinsam mit $200 \mathrm{~mL}$ Aceton kokondensiert. Nach Erwärmen des Kokondensates auf $-78^{\circ} \mathrm{C}$ wird eine schwarze Suspension aus einem schwarzer Feststoff in einer farblosen Lösung erhalten. Nach Erwärmen auf Raumtemperatur wird die Lösung abfiltriert und nach Einengen des blassgelben Filtrates erhält man farblose nadelförmige Kristalle von $\mathrm{SnBr}_{2} \cdot 2 \mathrm{OC}_{3} \mathrm{H}_{6} 6$ (1.6 g; $\left.4.1 \mathrm{mmol}\right)$.

\footnotetext{
${ }^{1}$ H-NMR (400 MHz, Aceton-d $\left.\mathrm{d}_{6}\right): \delta=2.03\left(\mathrm{~s}, 6 \mathrm{H}, \mathrm{CH}_{3}\right) .{ }^{13}$ C-NMR: $\left(100 \mathrm{MHz}\right.$, Aceton- $\left.\mathrm{d}_{6}\right): \delta$ $=30.58 \mathrm{CH}_{3}, 207.6 \mathrm{CO} .{ }^{119}$ Sn-NMR $\left(150 \mathrm{MHz}\right.$, Aceton-d $\left.\mathrm{d}_{6}\right): \delta=-67.53$.
}

\section{Synthese von $\mathrm{SnBr}_{2} \cdot 2 \mathrm{OC}_{4} \mathrm{H}_{8} 7$}

Es werden innerhalb von 2 Stunden $20 \mathrm{mmol}$ Bromwasserstoff bei $1240^{\circ} \mathrm{C}$ über metallisches Zinn geleitet, wobei $2.05 \mathrm{~g}(17 \mathrm{mmol})$ Zinn verbraucht werden. Die gasförmigen Produkte werden zusammen mit 200 mL eines Lösemittelgemisches aus Toluol und THF im Verhältnis von 4:1 bei $-196^{\circ} \mathrm{C}$ kokondensiert. Nach Erwärmen auf $-78^{\circ} \mathrm{C}$ wird eine schwarze Suspension 
aus einem schwarzer Feststoff in einer farblosen Lösung erhalten. Nach Erwärmen auf Raumtemperatur wird die überstehende Lösung abfiltriert und das Lösemittel unter vermindertem Druck entfernt. Der verbleibende weiße Rückstand wird in THF gelöst, woraus sich farblose Nadeln von $\mathrm{SnBr}_{2} \cdot 2 \mathrm{OC}_{4} \mathrm{H}_{8} 7$ bilden $(2.5 \mathrm{~g}, 5.9 \mathrm{mmol})$.

${ }^{1}$ H-NMR (400 MHz, THF-d 8 ): $\delta=1.75\left(\mathrm{~d}, J=6.7 \mathrm{~Hz}, 8 \mathrm{H}, \mathrm{OCH}_{2}\right) ; 3.61(\mathrm{t}, J=6.7 \mathrm{~Hz}, 8 \mathrm{H}$, $\left.\mathrm{CH}_{2}\right) \cdot{ }^{13}$ C-NMR: $\left(100 \mathrm{MHz}, \mathrm{THF}-\mathrm{d}_{8}\right): \delta=23.69\left(\mathrm{CH}_{2}\right), 65.88\left(\mathrm{OCH}_{2}\right) \cdot{ }^{119} \mathrm{Sn}-\mathbf{N M R}$ $\left(150 \mathrm{THF}-\mathrm{d}_{8}\right): \delta=-86.60$.

\section{Synthese einer SnBr-Lösung in $\mathrm{CH}_{2} \mathrm{Cl}_{2}$}

Es werden innerhalb von 2 Stunden $20 \mathrm{mmol}$ Bromwasserstoff bei $1240^{\circ} \mathrm{C}$ über metallisches Zinn geleitet, wobei $2.01 \mathrm{~g}(17 \mathrm{mmol})$ Zinn verbraucht werden. Die gasförmigen Produkte werden bei $-196^{\circ} \mathrm{C}$ mit insgesamt $200 \mathrm{~mL}$ eines Lösemittelgemisches aus Dichlormethan und Tributylamin im Verhältnis von 4:1 kokondensiert. Nach Erwärmen auf $-78^{\circ} \mathrm{C}$ erhält man eine dunkelbraune Lösung. $20 \mathrm{~mL}$ dieser Lösung werden in ein Schlenkgefäß überführt und auf Raumtemperatur erwärmt. Dabei bildet sich ein schwarzer Niederschlag (Abbildung 6 links) und man erhält eine blassgelbe Lösung.

Die restliche Lösung wird über zwei Wochen bei $-78^{\circ} \mathrm{C}$ gelagert, wobei die dunkelbraune Farbe immer mehr verblasst und man schlussendlich eine blassgelbe Lösung erhält. Erwärmen der blassgelben Lösung auf Raumtemperatur führt zu keiner weiteren Veränderung. Anschließend wird das Lösungsmittel im Vakuum entfernt, wobei ein hellgelber Rückstand (Abbildung 6 rechts) erhalten wird. 


\section{${ }^{119}$ Sn-Mößbauerspektroskopie}

Zur Aufnahme der ${ }^{119} \mathrm{Sn}-\mathrm{Mößbauer-Spektren} \mathrm{wurde} \mathrm{eine} \mathrm{Ca}^{119} \mathrm{SnO}_{3}$ Quelle verwendet. Die Messungen erfolgten in einer linearen Transmissionsanordnung. Die Proben wurden mit $\alpha-$ Quarz als Füllmittel in dünnwandige Glascontainer eingeschmolzen mit einer Belegung von etwa $10 \mathrm{mg} \mathrm{Sn} \cdot \mathrm{cm}^{-2}$. Zur Reduzierung der SnK-Röntgenstrahlung der Quelle wurde eine Palladiumfolie $(0.05 \mathrm{~mm})$ verwendet. Die Quelle wurde bei Raumtemperatur gehalten. Die Anpassung der Spektren erfolgte mit dem Programm NoRMOS-90. ${ }^{32}$

Tabelle 3: Anpassungsparameter der ${ }^{119} \mathrm{Sn}-$ Mößbauerspektren von $\mathrm{Sn}$ und der disproportionierten "Sn(I)Br”-Probe bei $77 \quad \mathrm{~K} . \quad \delta$ : Isomerieverschiebung; $\Delta E_{\mathrm{Q}}$ : Quadrupolaufspaltung; $\Gamma$ : experimentelle Linienbreite. Mit einem Stern markierte Parameter wurden während der Anpassung konstant gehalten.

\begin{tabular}{|c|c|c|c|c|}
\hline $\mathrm{T} / \mathrm{K}$ & $\delta / \mathrm{mms}^{-1}$ & $\Gamma / \mathrm{mms}^{-1}$ & $\Delta E_{\mathrm{Q}} / \mathrm{mms}^{-1}$ & Verhältnis \\
\hline \multicolumn{5}{|l|}{ Sn } \\
\hline 77 & $2.54(1)$ & $1.10(6)$ & $0.26(5)$ & - \\
\hline \multicolumn{5}{|c|}{ disproportionierte “Sn(I)Br"-Probe } \\
\hline \multirow[t]{3}{*}{77} & $0.08 *$ & $0.78 *$ & $0.49 *$ & 5.0 \\
\hline & $2.62(1)$ & $1.03(3)$ & $0.39(2)$ & 52.6 \\
\hline & $4.07(1)$ & $1.20(2)$ & $0.0^{*}$ & 42.4 \\
\hline
\end{tabular}


Tabelle 4: Kristallographische Daten und Details für die Strukturlösung von $\mathrm{SnBr}_{2} \cdot 2 \mathrm{OC}_{3} \mathrm{H}_{6} 6$ und $\mathrm{SnBr}_{2} \cdot 2 \mathrm{OC}_{4} \mathrm{H}_{8} 7$

\begin{tabular}{|c|c|c|}
\hline Summenformel & $\mathrm{SnBr}_{2} \mathrm{O}_{2} \mathrm{C}_{6} \mathrm{H}_{12} 6$ & $\mathrm{SnBr}_{2} \mathrm{O}_{2} \mathrm{C}_{8} \mathrm{H}_{16} 7$ \\
\hline Kristallsystem & Monoklin & Monoklin \\
\hline Raumgruppe & $\mathrm{P} 2{ }_{1} / \mathrm{n}$ & $\mathrm{P} 2{ }_{1} / \mathrm{c}$ \\
\hline Gitterparameter $\left[\AA ̊\right.$ bzw. $\left.{ }^{\circ}\right]$ & $\begin{array}{l}\mathrm{a}=9.1966(18) ; \mathrm{b}= \\
9.3218(19) ; \mathrm{c}=13.358(3) ; \\
\beta=90.41(3)\end{array}$ & $\begin{array}{l}\mathrm{a}=8.5419(16) ; \mathrm{b}= \\
10.1825(6) ; \mathrm{c}=15.0514(11) ; \\
\beta=104.662(6)\end{array}$ \\
\hline Volumen der Elementarzelle $\left[\AA^{3}\right]$ & $1145.2(4)$ & $1266.51(15)$ \\
\hline Formeleinheiten $\mathrm{Z}$ & 4 & 4 \\
\hline röntgenographische Dichte $\left[\mathrm{g} \cdot \mathrm{cm}^{-3}\right]$ & 2.289 & 2.217 \\
\hline Absorptionskoeffizient $\mu\left[\mathrm{mm}^{-1}\right]$ & 9.170 & 8.299 \\
\hline $\mathrm{F}(000)$ & 736 & 800 \\
\hline Kristallabmessungen $[\mathrm{mm}]$ & $0.5 \times 0.4 \times 0.3$ & $0.5 \times 0.4 \times 0.4$ \\
\hline Diffraktometer & STOE IPDS & STOE IPDS \\
\hline Messtemperatur $[\mathrm{K}]$ & 150 & 190 \\
\hline Wellenlänge $[\AA]$ & 0.71073 & 0.71073 \\
\hline Winkelbereich der Datensammlung $\left[^{\circ}\right]$ & $2.66-27.07$ & $2.44-27.12$ \\
\hline hkl-Bereich & $\begin{array}{l}-11 \leq \mathrm{h} \leq 11,-11 \leq \mathrm{k} \leq 10 \\
-17 \leq 1 \leq 16\end{array}$ & $\begin{array}{l}-10 \leq \mathrm{h} \leq 10,-12 \leq \mathrm{k} \leq 13 \\
-19 \leq 1 \leq 19\end{array}$ \\
\hline Zahl der gemessenen Reflexe & 8764 & 13251 \\
\hline Zahl der unabhängigen Reflexe & 2500 & 2774 \\
\hline beobachtete Reflexe mit $\mathrm{F}^{2}>2 \sigma$ & 2235 & 2592 \\
\hline Verwendete Rechenprogramme & SHELXL und SHELXS & SHELXL und SHELXS \\
\hline Zahl der verfeinerten Parameter & 108 & 155 \\
\hline $\mathrm{R}-$ Werte $[\mathrm{I}>2 \sigma(\mathrm{I})]$ & 0.0367 & 0.0338 \\
\hline $\mathrm{R}$ - Werte (alle Daten) & 0.1056 & 0.0848 \\
\hline Restelektronendichte min./max. $\left[\mathrm{e} \AA^{-3}\right]$ & 1.081 und -1.550 & 0.798 und -1.010 \\
\hline CCDC Hinterlegungsnummer ${ }^{33}$ & 726879 & 726880 \\
\hline
\end{tabular}




\section{Literatur und Fußnoten}

${ }^{1}$ A. Schnepf, Angew. Chem. 2004, 116, 680 - 682; Angew. Chem. Int. Ed. 2004, 43, $664-$ 666; A. Schnepf, Coord. Chem. Rev. 2006, 250, 2758 - 2770.

${ }^{2}$ H. Schnöckel, Dalton Trans. 2005, 19, $3131-3136$.

${ }^{3}$ A. Schnepf, Eur. J. Inorg. Chem. 2008, $1007-1018$.

${ }^{4}$ A. Schnepf, C. Schenk, Angew. Chem. 2006, 118, 5499 - 5502; Angew. Chem. Int. Ed. 2006, $45,5373-5376$.

${ }^{5}$ C. Schenk, A. Schnepf, Chem. Commun. 2008, $4643-4645$.

${ }^{6}$ A. Schnepf, Phosphorus, Sulfur and Silicon and the Related Elements, 2004, 179, 695 - 698.

${ }^{7}$ A. Schnepf, Chem. Soc. Rev. 2007, 36, $745-758$.

${ }^{8}$ a.) B. E. Eichler, P. P. Power, Angew. Chem. 2001, 113, 818 - 819; Angew. Chem. Int. Ed. 2001, 40, 796 - 797; b.) A. F. Richards, B. E. Eichler, M. Brynda, M. M. Olmstead, P. P. Power, Angew. Chem. 2005, 117, 2602 - 2605; Angew. Chem. Int. Ed. 2005, 44, 2546 2548; c.) M. Brynda, R. Herber, P. B. Hitchcock, M. F. Lappert, I. Nowik, P. P. Power, A. V. Protchenko, A. Ruzicka, J. Steiner, Angew. Chem. 2006, 118, 4439 - 4443; Angew. Chem. Int. Ed. 2006, 45, 4333 - 4337; d.) E. Rivard, J. Steiner, J. C. Fettinger, J. R. Giuliani, M. P. Augustine, P. P. Power, Chem. Commun. 2007, 4919 - 4921.

${ }^{9}$ G. Prabusankar, A. Kempter, C. Gemel, M.-K. Schröter, R. A. Fischer, Angew. Chem. 2008, 120, 7344 - 7347; Angew. Chem. Int. Ed. 2008, 47, $7234-7237$.

${ }^{10}$ Holleman-Wiberg, Lehrbuch der Anorganischen Chemie, 102. Auflage, Walter de Gruyter, Berlin, 2007, 1012.

${ }^{11}$ K. Balasubramanian, J. Molecular Spectr. 1988, 132, $280-283$.

${ }^{12}$ M. Häser, R. Ahlrichs, J. Comput. Chem. 1988, 10, $104-111$.

${ }^{13}$ F. Weigend, M. Häser, Theor. Chem. Acc. 1997, 97, $331-340$.

${ }^{14}$ F. Weigend, M. Häser, H. Patzelt, R. Ahlrichs, Chem. Phys. Lett. 1998, 294, 143 - 152. 
${ }^{15}$ F. Weigend, R. Ahlrichs, Phys. Chem. Chem. Phys. 2005, 7, $3297-3305$.

${ }^{16}$ C. Hättig, Phys. Chem. Chem. Phys. 2005, 7, 59-66.

${ }^{17}$ R. Ahlrichs, M. Bär, M. Häser, H. Horn, C. Kölmel, Chem. Phys. Lett. 1989, 162, 165 169.

${ }^{18}$ H. Saal, E. Milke, C. Brünig, M. Binnewies, R. Köppe, Eur. J. Inorg. Chem. 2008, 4146 4151.

${ }^{19}$ K.A. Gingerich, in E. Kaldis (ed.), Current Topics in Materials Science, 1980, 6, 345 - 462.

${ }^{20}$ M. B. Monagan, K. O. Geddes, K. M. Heal, G. Labahn, S. M. Vorkoetter, J. McCarron, P. DeMarco, Maple 10 Programming Guide, Maplesoft, Waterloo ON, Canada, 2005.

${ }^{21}$ M. Binnewies, E. Milke, Thermochemical Data of Elements and Compounds, Wiley, VCH, Weinheim, $2^{\text {nd }}$ Ed., 2002.

${ }^{22}$ M. Binnewies, Chemische Gleichgewichte, Verlag Chemie, Weinheim 1996.

${ }^{23}$ Der zur Berechnung angenommene Druck von $10^{-2}$ mbar entspricht dabei dem Arbeitsdruck innerhalb des Graphitreaktors während der präparativen Kokondensation.

${ }^{24}$ R. Köppe, A. Schnepf, Z. Anorg. All. Chem. 2002, 628, $2914-2918$.

${ }^{25}$ Eine weitere Erhöhung der Reaktionstemperatur auf $1270^{\circ} \mathrm{C}$ führt zu einer Veränderung des Zinn:Halogen Verhältnisses zu Sn:Br $=1: 0.95$, was darauf hindeutet, dass ab dieser Temperatur der Dampfdruck des elementaren Zinns nicht mehr vernachlässigbar ist und man während der Reaktion neben $\mathrm{SnBr}$ auch elementares Zinn verdampft.

${ }^{26}$ E. Kuzmann, T. Kégl, L. Kollár, A. Vértes, V. Kumar Garg, J. Radioanalytical Nucl. Chem., 1997, 220, $103-104$.

${ }^{27}$ N. N. Greenwood, A. Timnick, J. Chem. Soc. (A), 1971, 676 - 678.

${ }^{28}$ P. E. Lippens, Phys. Rev. B, 1999, 60, $4576-4586$.

${ }^{29}$ P. J. Smith, L. Smith, Inorg. Chim. Acta 1973, 7, 11 - 16. 
${ }^{30}$ A. Schnepf, Eur. J. Inorg. Chem. 2005, 2120-2123.

${ }^{31}$ A. Schnepf, Habilitationsschrift „Erzeugung gasförmiger Ge(I) Halogenide bei $1600^{\circ} \mathrm{C}$ und deren Einsatz in der Synthesechemie "Karlsruhe 2006.

${ }^{32}$ R. A. Brand, Normos Mössbauer fitting Program, Universität Duisburg, 2002.

${ }^{33}$ Weitere Informationen zur Kristallstrukturuntersuchung können beim Cambridge Crystallographic Data Centre (CCDC, The Director, 12 Union Road, Cambridge, CB2 1

EZ, U.K.; FAX +49-1223-336033; E-mail: deposit@ccdc.cam.ac.uk bzw.www: http://www.ccdc.cam.ac.uk) unter der Hinterlegungsnummer CCDC 726879 6, CCDC 7268807 angefordert werden. 